\title{
Supporting Ethnographic Studies of Ubiquitous Computing in the Wild
}

\author{
Andy Crabtree, Steve Benford, Chris \\ Greenhalgh \\ School of Computer Science \& IT \\ University of Nottingham, Jubilee Campus, \\ Wollaton Road, Nottingham NG8 1BB, UK. \\ $\{$ axc, sdb, cmg\}@cs.nott.ac.uk
}

\begin{abstract}
Ethnography has become a staple feature of IT research over the last twenty years, shaping our understanding of the social character of computing systems and informing their design in a wide variety of settings. The emergence of ubiquitous computing raises new challenges for ethnography however, distributing interaction across a burgeoning array of small, mobile devices and online environments which exploit invisible sensing systems. Understanding interaction requires ethnographers to reconcile interactions that are, for example, distributed across devices on the street with online interactions in order to assemble coherent understandings of the social character and purchase of ubiquitous computing systems. We draw upon four recent studies to show how ethnographers are replaying system recordings of interaction alongside existing resources such as video recordings to do this and identify key challenges that need to be met to support ethnographic study of ubiquitous computing in the wild.
\end{abstract}

\section{Author Keywords}

Ethnography, ubiquitous computing, record and replay.

\section{ACM Classification Keywords}

C.3 Special-purpose and Application-based Systems.

\section{INTRODUCTION}

With its focus on the situated nature of interaction and the social character of use this reveals [28], ethnography is especially relevant to understanding the purchase of ubiquitous computing in a wide variety of social settings. Ubiquitous computing is explicitly concerned with the setting in which interaction occurs and how this may be exploited to shape user experiences, for example developing context-aware technologies that are able to

\section{COPYRIGHT NOTICE}

\author{
Paul Tennent, Matthew Chalmers, Barry Brown \\ Department of Computing Science \\ University of Glasgow \\ 17 Lilybank Gardens, Glasgow G12 8QQ \\ United Kingdom \\ $\{$ pt, matthew, barry\}@dcs.gla.ac.uk
}

dynamically adapt to different users, places and activities. As these and other ubiquitous technologies mature it is important to compliment their design with a thorough understanding of their impact on situated action in the wild in order to ensure that they resonate with the social circumstances of their use. Exploiting ethnography to evaluate how ubiquitous applications are 'made to work' in situ is a useful means of understanding the adaptations that are required to make new technologies 'fit' complex arrangements of real world, real time activity [30].

The study of ubiquitous computing in the wild raises new challenges for ethnography. The advent of invisible sensing systems now allows a novel range of new experiences to be constructed and deployed in the wild [e.g., 14, 4, 6]. It also means that ubiquitous computing distributes interaction across a burgeoning array of different applications and devices, some online, some mobile, each exploiting different mechanisms of interaction. If ethnographers are to develop coherent understandings of interaction in changing circumstances of design it is necessary that they reconcile the various 'pieces in the game' as it were. This requires ethnographers to supplement traditional resources external to the digital setting of interaction, such as audio and video recordings of action and talk, with resources internal to the digital setting, such as the text messages and audio files generated by users in their interactions together [2].

This paper extends interdisciplinary research concerned to support qualitative studies of technology [25, 31, 7]. We review four recent studies to show how ethnographers have responded to the challenge by exploiting system recordings of digital events and replaying them alongside external resources, particularly video, to provide richer portraits and understandings of interaction within ubiquitous environments. The primary purpose of the paper is to articulate the development of 'record and replay' tools that instrument ubicomp environments to support ethnographic study, and to reflect on experiences of using them to inform the development of new tools in the future. Ultimately our aim is foster a program of research and development that incorporates ethnography into ubiquitous computing by design, exploiting the inherent features of ubiquitous computing applications to complement existing techniques of observation, data production, and analysis. 


\section{WHAT DO WE MEAN BY 'ETHNOGRAPHY'?}

We ask the question because ethnography is a gloss on heterogeneous practices and ways of thinking and because we think it important to situate our work in its proper context in order that both our approach to ethnography and technological support not be misconstrued. We have already suggested that ethnography is concerned to understand situated action, but this is not the concern of a great many ethnographers in the social sciences. Rather, it is the concern of ethnographers working in the ethnomethodological (EM) tradition [17]. EM does not consider ethnography to be a specialized way of looking at the world. On the contrary, ethnography is the most mundane of things from this perspective, something we all do as ordinary members of society situated as we are in the various settings in which we live out our daily lives.

... the member "in the midst" of witnessed actual settings recognizes that witnessed settings have an accomplished sense, an accomplished facticity, an accomplished accountability ... That accomplishment consists of members doing, recognizing, and using ethnographies. Harold Garfinkel [15]

Ethnographies are, to use a phrase, a 'seen but unnoticed' feature of everyday life. When we go to work and ask of our colleagues "what's happening today?", we are doing an ethnography. When we go to the hardware store and seek out just the right materials for a DIY project, we are doing an ethnography. When we sit down for dinner and ask our family or friends about their day, we are doing an ethnography. Ethnographies permeate every aspect of our ordinary lives in the most passing and unremarkable of ways. So if ethnography is a ubiquitous feature of everyday life why is it considered (by some at least) to be a sound approach to social science research - and one that is of relevance to IT research and systems design?

A distinction may be made between lay and professional ethnographies, where a recognizable difference exists as to the ways in which ethnographic materials are analyzed. It is not that in our capacity as ordinary members we do not analyze ethnographic materials - clearly an account of what's happening at work or a family member's account of his or her day is open to analysis - but that we do so in different ways to professional ethnographers. It would be very strange, indeed fractious, if you were to start classifying a family member's descriptions of her day in terms of formal analytic schema in order to analyse it, for example, yet that is what a social science researcher might well do with such ethnographic materials [15]. ${ }^{1}$

1 Of course, it might be argued that professional ethnographers observe and gather data as well as analyse it but there is nothing special or privileged in the observing or gathering of data, ordinary members do it too: there is nothing intrinsically different about visiting a novel setting, seeing what goes on there, asking questions, making notes or diagrams or videos, etc. What is distinctly different is the way in which the 'stuff' observed and gathered is analysed.
There are two basic features that mark professional analyses out as distinct. One is the documentary character of professional analyses: professional analyses come packaged in reports. Secondly, there are two fundamental ways in which ethnographic materials are 'reported' or textually rendered: 1) Through formal analysis, which renders ethnographic materials in terms of coding schemes, taxonomies, grand theories or narratives, models and other situationally absent descriptions [16]. 2) Ethnomethodologically, through thick description of the practical action and practical reasoning exhibited by members in the unfolding course of their activities together [28]. ${ }^{2}$ Both approaches recognize that analysis is intimately bound up with description; that the way in which you describe something is, to use another phrase, 'reflexively' connected to your analysis [27]. In producing ethnographic texts, both formal analysis and ethnomethodology have an abiding concern with reflexivity, but in very different ways.

Formal analysis is largely - but by no means exclusively concerned with 'analytic reflexivity', a notion which came into focus following the crisis of representation that marked the post-modern turn in the social sciences [23]. With analytic reflexivity attention is directed towards critical self-reflection in order to understand the ways in which the very act of ethnography and ethnographic reportage shapes our understanding of a setting, and towards finding solutions to the professional belief that there is an inevitable degree of cultural and subjective bias built into the act [24]. Accepting the tradition and good sense of critical reflection EM nevertheless rejects what Bittner [8] refers to as the "self-indulgent concentration" of analytic reflexivity, and thus replaces a "pallid ideology of cultural relativism" with a concern to appreciate the 'reflexivity of accounts' [15].

The reflexivity of accounts directs attention away from the analyst and formal representations towards the everyday settings that people inhabit and to the observable and reportable accounting practices - or 'work-practices' as they are often called - that populate, illuminate and shape those settings [13, 17]. Such 'ethnomethodologically-informed ethnographic studies' played a fundamental role as IT research turned to the social in late 80's following Lucy Suchman's seminal work on the problem of humanmachine communication [29]. They continue to be exploited to inform systems design and today 'ethnographies', as such studies are often if somewhat misleadingly referred to, are an accepted feature of IT research and systems design. Indeed, a corpus of such studies now populates the HCI, CSCW and DIS literature, elaborating the accountable character of interaction and collaborative work, of organization, of technology use in the workplace, and, as design diversifies, of other settings in everyday life as well.

\footnotetext{
2 That is thick description pace Gilbert Ryle [26] who coined the phrase, but not Clifford Geertz [18] who popularized it and made it answerable to formal analysis.
} 


\section{A NEW CHALLENGE FOR ETHNOGRAPHY}

The use of ethnomethodologically-informed ethnography or ethnography as it will simply be called from here on in is not without its troubles in contemporary IT research. The continued diversification of computing in everyday life, and the emergence of ubiquitous computing in particular, raises new challenges for ethnography. The following issues are of particular concern.

- Users of ubiquitous systems are often mobile. They move across extended physical areas, quickly at times, sometimes even running, which can make it difficult to document action and capture video material.

- Interaction often involves the use of small displays, such as handheld computers and mobile phones. This makes it difficult to see users' interactions with the system. Users may also use headphones, so little of the talk between participants is available.

- Users often interact with invisible sensing systems, for example, Global Positioning Systems or video-tracking, which means that it can be difficult to understand why users are acting in a given way and how the sensing systems are behaving.

- Interaction is distributed across different applications and devices. Interaction is not only located in different physical locations and online but is also mediated through different applications and devices (such as PDAs and virtual environments), which makes it difficult to develop a coherent description of interaction.

In short, describing the accountable interactional character of ubiquitous applications in the wild is different to describing more traditional kinds of computing environment, where an ethnographer can document and record interaction at various points in the division of labour and build up a coherent description of the organization of action in a setting and the use of technology therein [e.g., 20]. In ubiquitous environments interaction has a much more invisible and fragmented character however, involving a greater array of applications and devices in the user experience. The problem is one of reconciling the fragments - for example, reconciling what happens on the streets via GPS-enabled PDAs, with what happens online via virtual models, with what ways interaction is articulated between the two (e.g., via audio on the one hand and text messages on the other).

Ubiquitous systems move beyond desktop PCs and their variants, and beyond telephones and paper, to introduce a new level of complexity into ethnographic study which warrants the development of new tools to support thick description, and with it evaluation, in this burgeoning domain. The need to develop applications to support social science researchers in this area has already been recognized by HCI researchers. For example, Woodruff et al. [31] and Brown et al. [7] have exploited the logs from electronic guidebooks and sensors to thicken ethnographic and conversation analytic descriptions of interaction. There is of course nothing new in exploiting systems logs to understand interaction. HCI researchers have been exploiting system logs for decades and techniques for doing so are documented in most good HCI textbooks. More recent efforts have sought to automate usability evaluation $[19,21]$ and HCI researchers have recently sought to extend current approaches to support qualitative research [22].

Ubiquitous computing goes beyond logging machine states and events however, to record elements of social interaction and collaboration conducted and achieved through the use of ubiquitous applications as well. Thus, and for example, audio and text messages are logged alongside machine states and other events. System recordings make a range of digital media used in and effecting interaction available as resources for the ethnographer to exploit and understand the distinctive elements of ubiquitous computing and their impact on interaction. The challenge, then, is one of combining external resources gathered by the ethnographer with a burgeoning array of internal resources to support thick description of the accountable character of interaction in complex digital environments.

\section{RESPONDING TO THE CHALLENGE: SOME EARLY EXPERIENCES}

Recent ethnographic studies of ubiquitous computing applications have begun to respond to the challenge by augmenting video data with system recordings. To varying degrees system recordings enable ethnographers to replay users' interactions within the system and to build up thick descriptions of the accountable character of interaction in ubiquitous computing environments, where interaction is distributed across a multiplicity of applications and devices. Below we recount salient features from four experiences of conducting ethnographic studies using a combination of video material and system logs. Between them, these experiences, which are presented in chronological order, demonstrate the increasing use of system logs to uncover the subtleties of interaction and collaboration. They reveal core challenges of this approach and introduce some prototype tools to help the ethnographer capture, manage and review combinations of system logs and videos. Our aim in discussing these experiences is to highlight emerging practice and to raise requirements for future tools to support ethnographic study in this challenging domain.

\section{Experience 1. Can You See Me Now?}

The first experience, Can You See Me Now? (CYSMN), is a ubiquitous game in which online players logged on over the Internet are chased through a virtual model of a city by professional performers who, equipped with handheld computers, GPS receivers and $\mathrm{WiFi}$, have to run through the actual city streets in order to catch them [14]. Online players collaborate by sending text messages to performers and to one another, and performers collaborate using walkie-talkies, with a digitized version of this audio being streamed to the online players. 


\section{Observing Interaction in CYSMN}

Studying interaction in CYSMN proved challenging due to the highly distributed and mobile nature of the participants. Remote online players could not be directly observed and the performers were spread across the game zone (of approx. $1 \mathrm{sq} \mathrm{km}$ ) and were moving around it quickly. Description and analysis thus exploited a combination of several hours of video material of the performers' activities on the streets and system logs of online players' interactions with the game server. The system logs consisted of time-stamped records of all participants' interactions with the game server, including their movements (resulting from key presses for online players and GPS updates for performers), text messages (from online players), and significant game events (capture events). Separate logs were kept of raw GPS data, and network traffic from the tcpdump tool.

\section{Description and Analysis of CYSMN}

Description and analysis of CYSMN exploited several resources. Visualizations of the GPS and WiFi logs yielded a general picture of how these technologies had performed during the experience, most notably revealing the considerable inaccuracy of GPS and limited coverage of both GPS and WiFi throughout. Transcription and analysis of video of the performers revealed how the uncertainties produced through variable GPS and WiFi coverage had a major impact on interaction and required the runners to devise diagnostic practices to identify and resolve the troubles caused by poor GPS and/or WiFi coverage [9].

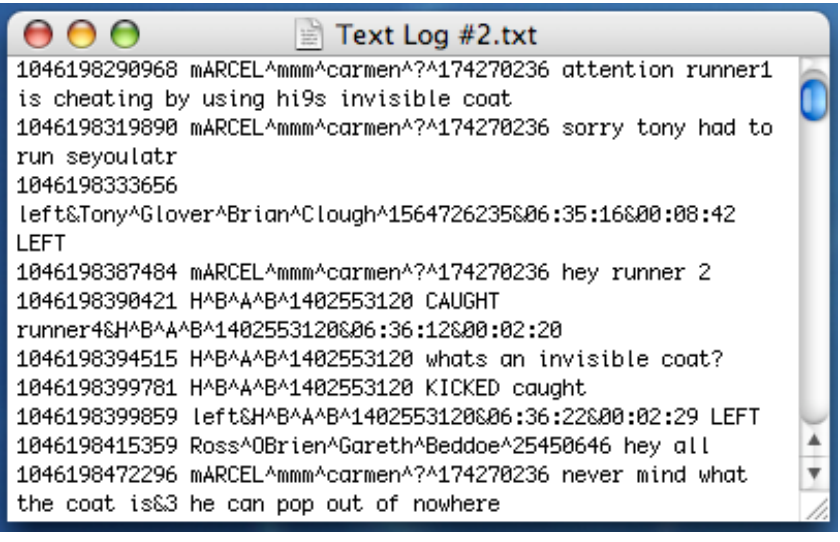

Figure 1. The raw text log from CYSMN.

However, given a lack of video data for online players, the ethnographer needed to turn to a different source in order to understand their experiences of these troubles: the text message logs (Figure 1). Examining these logs posed its own problems. Not only were they full of obscure characters, difficult to read and marked by a multiplicity of overlapping conversational threads, more importantly, they could not be replayed alongside sequences of video to explore the immediate interactional relationship between runners and players.

In part this was due to the lack of a suitable log replay tool at the time, but also because the log itself associated just a single moment in time with each message, i.e., when it passed through the server. To understand the experience in detail richer timing information was required, for example the period over which the text was typed, the times at which the message became visible on different displays, and outside the scope of system logging - the times at which particular participants noticed and read the message. These logs were useful, however, and analysis of online players' messages to one another and to the runners yielded insights into the ways in which they organized collaboration and managed the uncertainties caused by technical troubles as a feature of their collaborative activities $[2,5,10]$.

\section{Issues Raised by CYSMN}

System logs provided a useful way of recording and understanding the interaction between online players and something of their relationship to the runners on the ground. These logs made the experience available at quite a different level of granularity to video recordings, however. Video provided a very detailed picture but only for limited parts of the overall experience (i.e., a short period of the runners' experience), whereas $\operatorname{logs}$ were available for across the experience (some 6 days) but offered a less detailed and coherent view of events.

For this reason our analysis of interaction between runners and online players in CYSMN was at a fairly broad level, pulling out broad organizational features of the experience but stopping short of analyzing moment-by-moment interactions between online players and runners, such as the collaborative character of particular chase and capture sequences. Overall, the experience of using text logs to observe, describe and analyze interaction in CYSMN highlighted the need to combine a richer set of resources that would enable closer inspection of the relationship between online and mobile participants.

\section{Experience 2. Uncle Roy All Around You}

Uncle Roy All Around You (URAY) was a follow on project to CYSMN that also explored the idea of connecting a physical city to a parallel online 3D model of a city. This time, however, players were on the streets as well as online and involved in searching the city for a mysterious character called Uncle Roy, following a series of locationbased clues provided by the game and being guided by online players in the effort to find him. Street players were also covertly shadowed by performers to aid them if they went of track or if technical troubles interfered with gameplay. Communication between street and online players involved the street players recording and uploading short audio messages and online players replying with text messages. GPS positioning was replaced with the low-tech but more reliable solution of self-reported positioning where street players declared their positions in the game through their use of an electronic map. Like CYSMN, URAY was played in an urban space of about $1 \mathrm{sq} \mathrm{km}$ for several hours a day for one or two weeks in each of the three cities it has toured to date. Several thousand players 
have so far taken part, with up to ten street players and fifteen online players being active at any one time.

\section{Observing Interaction in URAY}

Ethnographic study of URAY provided an opportunity to further develop the approach of exploiting video and system recordings in order to construct thick descriptions and analyses of interaction. Video recordings were made of twelve street players, from over two hundred who took part in the first experience, and consisted of following their journey through the city and recording their interactions. Again, system logs of all interactions as seen by the central server including self-declared positions, game events (such as triggering clues), text messages, and audio messages were recorded.

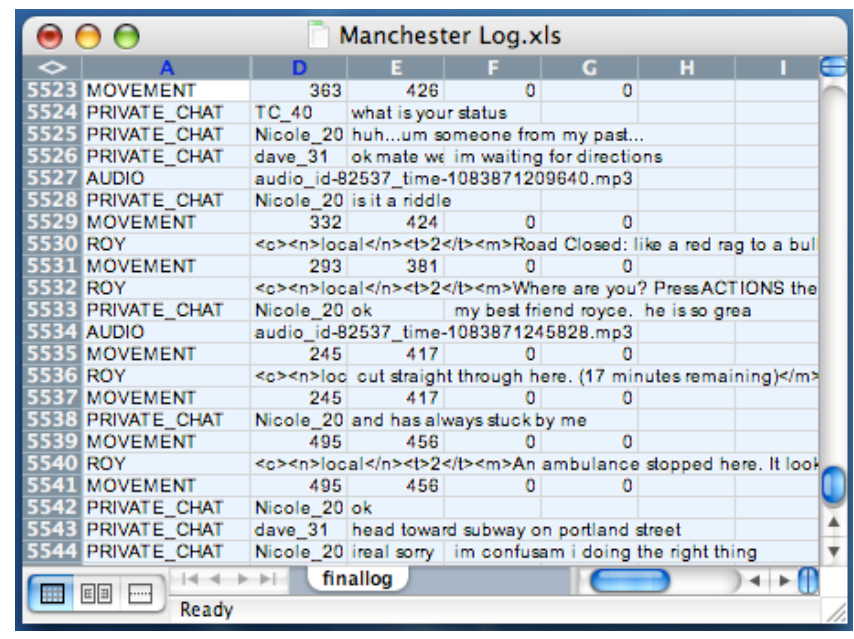

Figure 2. The raw log from URAY.

\section{Description and Analysis of URAY}

Description and analysis of URAY exploited all of these resources. Visualizations of self-reported positions revealed distinct patterns of error between real and reported position [3] and subsequent ethnographic analysis sought to unpack the social character of these errors [11]. Text messages and clues were provided in a temporally ordered spreadsheet with audio files being supplied separately and indexed in the $\log$ by player ID and time (Figure 2). The text messages, clues and audio files were analyzed alongside video recordings to develop rich and detailed descriptions of player's actions on the streets and collaborations with online players.

Using the logs required the ethnographer to tease out the conversational threads that related to the sequences of interaction recorded on video from the text $\log$, to transcribe the contents of the audio files, and to integrate these with the contents of the video, thereby producing through manual manipulation thick descriptions of interaction occurring online and on the streets [12]. Analysis revealed multiple sources of error, some technical, others 'interpretative' which arose from street players practices of embedding the technology in the streets, following clues, and making sense of online players instructions [4]. Thus it was revealed that it was not only technical disconnections that lead players astray but also practices of use and collaboration, and these insights informed the design of an orchestration interface to support the management of errors by behind-the-scenes staff.

\section{Issues Raised by URAY}

Recording the resources exploited by participants in their digital collaborations made it possible to combine them with video and to describe and analyse interaction as it unfolded. While it took a considerable amount of manual work to interleave the transcribed contents of internal resources (text messages, audio files and clues) and external resources (video), the system logs in URAY enable the ethnographer to go beyond separate analyses of interaction on the streets and interaction online to examine the relationship between the two. Thus it was possible to observe, describe and analyse particular sequences of interaction between street players and online players and to unpack its accountable character [11]. While advancing support for ethnographic analysis, the manual character of $\log$ use raised the issue of developing tools that would enable the ethnographer to automatically replay and exploit the resources they provide. URAY also demonstrated the need to be able to focus on particular threads within the overall experience, such as a particular street player, and the varying online players with whom they interact as the experience unfolds. As ubiquitous computing scales up to bigger experiences and more users this becomes increasingly important.

\section{Experience 3. Savannah}

Savannah is an educational game in which children learn about the ecology of the African savannah, specifically about lion behaviour. Groups of six children at a time roleplay being lions by exploring a virtual savannah that appears to be overlaid on an empty school playing field, an open grassy area of roughly ninety by sixty metres. Equipped with handheld computers with WiFi networking and GPS location sensing, the children move around the playing field, exploring the varied terrain of the savannah and discovering the resources that lions need to survive. A series of trials of Savannah were conducted at a school over a period of three days during which time it was played by six groups of children.

\section{Observing Interaction in Savannah}

Savannah was more constrained in space and time than the previous two experiences, and it was possible to capture video for most of the experience using two cameras located just off the field of play. Each camera followed one player throughout, using pan and zoom to keep them in shot, with wireless microphones being used to capture their conversations with other nearby players. Even so, details such as the screens of the handheld computers were not visible. System logs of all game actions (GPS triggered movement updates, interactions such as attacking animals and game messages) were captured at a central server. 


\section{Description and Analysis of Savannah}

For Savannah we developed a dedicated game replay interface, which enabled the analyst to view the system logs in a range of ways [6]. A bird's eye view of the virtual savannah showed the positions of content such as animals (specified as discretely bounded colored regions called locales) and also the moment-by-moment positions of the six lions (as reported by GPS). This could also be overlaid with the positions of key past and future events such as 'attacks' and representations of the trails of different lions. Complete game status information was displayed for all players, including hunger, thirst and attack events. It was also possible to view what a selected player would have been seeing and hearing via their PDA at a given moment in time.

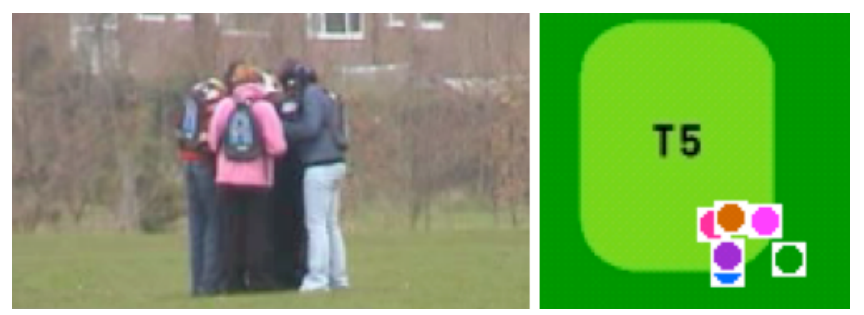

Figure 3. Exploiting video and system replay to understand interaction in Savannah.

The combined resources of a relatively comprehensive video record and the log visualizations available from this replay tool supported a finer-grained analysis of GPS sensing and location-based interaction than in the previous experiences. For example, initial inspection of the video revealed - through player dialogue within the experience that there were problems with groups of players being able to coordinate their actions, especially attacks on potential food. A step-by-step comparison of players' actions from the video alongside the system's view of events showed that attacking groups, while physically very close, would often span virtual locale boundaries, in part due to the tendency of players to immediately stop when their received new content (i.e., on crossing a boundary) and for other players to then gather around them (Figure 3).

\section{Issues Raised by Savannah}

Savannah demonstrated the utility of being able to replay and visualize system logs alongside video recordings in order to review how an experience unfolded from momentto-moment and to understand in detail how each participant was interacting with the system. However, powerful as this was, there were also some frustrations. Most notably, the clocks on the video cameras were not synchronized to one another or to the system clock used as the basis for the log files. The video and system replay interfaces were separate, unsynchronized and it was not possible to automatically cue one from the other. Furthermore, there were no facilities for check-pointing or otherwise marking sequences of interest with the replay itself. In short, while the interweaving of video and system records turned out to be very powerful, it was also unnecessarily difficult and time consuming for the analyst and highlighted the need for new tools that would better support the descriptive and analytic process.

\section{Experience 4. Treasure}

Treasure is a team-based, location-based, mobile game currently played by four players over a small area, typically around 100 square metres [1]. The game exploits the coverage of an 802.11 wireless network, and encourages players to learn about the boundaries and the effects of those boundaries upon one's ability to communicate with a server. 'Coins' are dropped on a map around the outside of a network, and players must carry GPS enabled handheld computers to the points indicated on the map to retrieve the coins, then carry them into an area of wireless network coverage and upload them to a server to receive points. However, when within network coverage opposing players can steal coins which have been picked-up but not uploaded.

\section{Observing Interaction in Treasure}

While connection and disconnection was an emergent issue of ethnographic study in CYSMN, it was an explicit aspect of design and game-play in Treasure. Consequently, to support this, system logs were recorded both on the central game server and on players' handheld computers, to capture the divergences expected to arise during periods of disconnection. Each handheld computer recorded GPS measurements, WiFi signal strength, current access point MAC address, the number of coins carried, GUI events (e.g., buttons pressed) and game events (such as instances of uploads and coin stealing). The server recorded its own view of each player's GPS, signal strength, score, and current coins, the current state of the network map (a WiFi coverage visualization which was a shared resource within the game itself), and all game events visible to the server. Additionally the server recorded a message log (all messages passed through the server). Video was recorded both from a roving camera in the game area which followed a single player, and from a window above the game area, which provided a broad view of the game area and also allowed us to zoom in on an individual player at any time.

Treasure also went beyond previous experiences in that the ethnographer was provided with a handheld computing running an in-house tool called QCCI (Quickie) to support observation and recording of interaction. This tool recorded the ethnographer's GPS position and subsequently allowed her to see where she had recorded audio and video on the combined map view. Additionally Quickie synchronized the clock on the handheld computer with that of the server and displayed a digital 'clapperboard' on the screen. The ethnographer could then use this to 'mark' or time-stamp their field recordings for subsequent re-synchronization with the system logs (e.g., by video recording the time display on the screen at the start of each video recording). An iPod was also available for the ethnographer to record audio notes in the field. 
As with Savannah, having data on several different devices (in this case the various handheld computers as well as the server) can make collection difficult. For Treasure, an automated FTP (file transfer) system was used whereby the players' handheld computers would automatically upload their logs to the server after the end of the game (at the request of the server).

\section{Description and Analysis of Treasure}

A dedicated system replay tool called 'Replayer' was created for integrating and reviewing all of the recorded log data, video and audio from each game (Figure 4). As with the Savannah replay tool, an active map of the game area visualizes players' movements, GPS and in this case network data. A searchable text-based amalgamation of the system logs is also displayed. In addition, multiple audio and video files can be opened simultaneously within the tool, and played back in synchrony with the map and text $\log$ views. The log search function allows the ethnographer to search for a particular kind of system event, jumping to the moment at which it occurred. The logs and video may then be played back and examined in detail.

This approach has helped to expose several interesting interactions in Treasure. For example, the positions recorded on the server for a player are often dramatically different from the position recorded by the GPS on the handled computer. This is primarily an effect of disconnection: when a player is outside the coverage of the network, she is unable to send position information to the server, and the server (and hence other players) has to work with the last-known position. Also, having the synchronized video streams allows us to observe GPS errors as discrepancies between map position (determined from logged GPS position) and actual position on the ground from video, helping to make more sense of the actions of players compared to system log or video evidence alone.

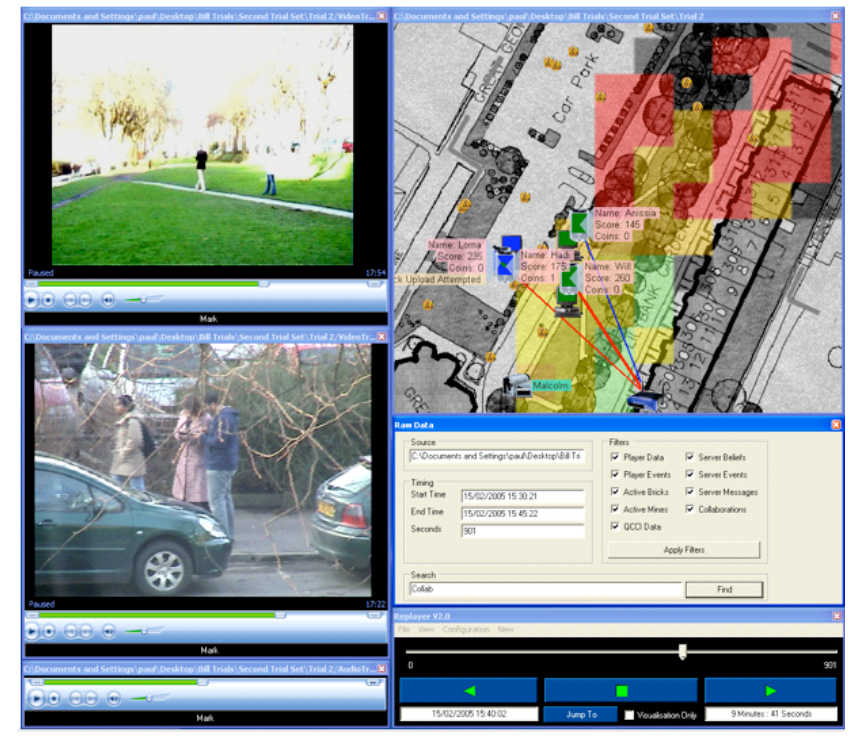

Figure 4. The Replayer interface integrating video replay and system log visualization and searching from Treasure

\section{Issues Raised By Treasure}

Quickie and Replayer demonstrate possible facilities to support ethnography, both during and after the event. Further design issues are also apparent however, particularly in relation to Replayer. This interface supports the synchronized juxtaposition of textual and map representations of system logs with video and audio field notes, but it is still rooted in a VCR metaphor of temporal navigation - a moving moment in time - whether this be by browsing or by searching for particular kinds of event.

While useful for real time purposes of inspection and analysis, this is still a long way from supporting the kind of documentary practices ethnographers typically exploit. In other words, tools are required to support the active transformation of replayed events into recognizable social science accounts. Furthermore, the use of Replayer also relies on technical knowledge of, e.g., the formats of system events and their internal names, and typically requires one of the system developers to be present during replay and analysis. This raises issues of how we might develop tools to more directly enable social science researchers to use record and replay tools themselves and it is towards addressing these and related issues that we now turn.

\section{REVISITING THE NEW CHALLENGE}

In presenting the case for record and replay we articulated a number of distinct aspects of ubiquitous computing environments that posed challenges for ethnographic study of the accountable character of interaction. Before moving on to consider the broader set of requirements that have emerged from our experiences, we briefly revisit these concerns to articulate the ways in which record and replay is enabling ethnographers to respond to them.

\section{Users of Ubiquitous Systems are often Mobile}

The level of mobility that we have observed in these examples challenges normal modes of field study. However, we have also seen that the ubiquitous computing technologies themselves, with their concern to support mobile users, can be directly exploited to support the process of description and analysis. In some cases, existing elements of the system - as recorded in system logs provide the necessary resources for study. In other cases, we might seek to add further facilities into the system to support description and analysis. For example, adding additional sensors (such as accelerometers) to handheld devices, or making more use of their built-in microphones and cameras would provide additional resources for analysis. However, this must be balanced with the impact of informed consent.

\section{Interaction often Involves the Use of Small Displays}

The replay interface to Savannah allowed the analyst to directly reconstruct views of the handheld computers' displays as she inspected the system logs. When coordinated with video footage, this can directly address the immediate concern of access to a participant's view and use of such small displays. As such this is a worthy ideal of any 
record and replay system, though realizing this is not without its problems. Not all interface-building technologies or platforms may admit this level of logging, for example, and the logs themselves may consume considerable space, and this on relatively small, lowpowered and limited-storage devices. Developing tools and techniques to support this kind of logging represents a real challenge for design.

\section{Users often Interact with Invisible Sensing Systems}

As we have seen, one of the primary uses of system logs in description and analysis is to gain access to sensed but otherwise invisible information, such as GPS-reported position or WiFi coverage. Also, as we have seen, these sensing systems are often imprecise and otherwise limited (e.g., in spatial coverage), and it is often only through finegrained comparison of visualizations of system logs with external records that these limitations - and users responses to them - become apparent. Record and replay is an invaluable resource in this area enabling ethnographers to understand the effects of sensing on interaction and to articulate the heterogeneous practices that users devise to organize and manage interaction in sensed environments.

\section{Interaction is Distributed across Different Applications and Devices}

Like mobility, distribution of interaction is both a characteristic challenge of ubiquitous computing and also a directly supported feature of the technologies themselves. Thus, the various system logs provide a rich resource for linking and relating otherwise fragmented interactions. However, care is required to correctly combine logs across such distributed systems (e.g., to synchronize them and to correctly identify corresponding events and entities). At the same time, however, we must be wary 'over-reconciling' distributed events, and this is a major concern for future requirements as we outline in the following section.

\section{REQUIREMENTS FOR FUTURE TOOLS}

Despite the benefits of 'record and replay', where the various digital resources implicated in interaction in ubiquitous environments are made available alongside external resources for the ethnographer, these studies and early prototype tools also highlight some outstanding challenges that need to be addressed.

\section{Distributed Logging and the Canonical System View}

In distributed ubiquitous applications, especially those that are based upon peer-to-peer rather than client-server architectures, or where there are multiple servers or extensive disconnection, system logging becomes far more complex than in traditional desktop applications. In such cases, there will be no readily available central view that can be taken to be in any sense as a 'canonical' representation of events or of the state of the system. Each user may have experienced a highly individual view, seeing events unfold in different ways and, depending on the system's internal data consistency policy, may perhaps be seeing quite different events. Such situations require a far more sophisticated approach to logging, collection and reconciliation of logs after the event.

Even where there is a single central server, it cannot always be assumed that this represents the canonical view of events. Network delays mean that different users and servers will have experienced system interactions and responses at different times and possibly in different orders. This affects fine-grained analysis in which the temporal order of interaction and talk is important [e.g., 31] and is an important factor to be reckoned with when the effects of delays or inconsistencies are a focus of interest [e.g., 9]. In such situations, ethnographers also require tools for visualizing and reasoning about the impact of delays, disconnections and divergence on each participant's view(s) of the system [12], both individually, and in relation to one another.

\section{Dealing with Larger Sets of Systems Logs and Video Recordings}

Different resources for analysis typically exhibit different patterns of availability, especially in an experience that runs for many hours, days, or weeks, or that involves many participants. System logs will often be available for most, if not all, of an experience, whereas video may only have been captured for just a few selected players at limited times, as we saw with CYSMN, Uncle Roy and Treasure. In order to construct thick descriptions of the accountable character of interaction based on an examination of system logs and video recordings, the ethnographer needs to first identify those periods and incidents for which both sets of resource are available. For example, a timeline-based visualization interface that enables the ethnographer to quickly review the availability of different resources at different times and so quickly select relevant episodes. Mechanisms such as this will support the effective combination of large resources and save considerable time and effort.

In turn, this needs to be supported by common formats and metadata for system and video recordings so that they can be more easily and consistently named, archived, indexed, reused and shared. While mundane, this has been a very real problem in our studies and one that is likely to become much more acute as the scale and complexity of studies increases. Similarly, while tools such as Quickie provide some support for synchronization between video and system recordings, these need extending to support more complex distributed studies that involve multiple media in interaction (not only spatial logs, but audio and text logs as well, for example).

\section{Designing for Ethnographic Practice}

The need to support ethnographic practice raises a further but interrelated set of issues which are of both immediate and generic concern and are tied to the work involved in producing thick descriptions. Experiences reported by 
ethnographers to date highlight four main issues in this area [12].

- Tools to support the identification of salient features to analysis in system recordings (e.g., conversational threads).

- Tools to support the extraction of salient features from system recordings so that discrete sequences of interaction may be described and analyzed.

- Tools to support the synchronization of internal and external resources so that they may not only be played side-by-side but be integrated as well.

- Tools to support the representation of social science data (e.g., annotation and transcription).

While tools such as Replayer enable the synchronization of internal and external resources, they do not support the integration of content from system recordings (such as text messages and audio files) and external recordings (video), into social science representations and accounts. This must be done manually at the current moment and outside of replay environments. Furthermore, the use of current facilities often requires the involvement of technical staff. In short, record and replay applications cannot yet be placed in the hands of the ethnographer. Not only is there a need to address the technical issues outlined above then, but there is a corresponding need to develop tools that are usable by ethnographers.

The 'usability' of the matter recognizes that ethnographic data, like all social science data, is an active construct. Data is not simply contained in system recordings but produced through their manipulation: through the identification of salient conversational threads in texts logs, for example, through the extraction of those threads, through the thickening up of those threads by synchronizing and integrating them with the contents of audio logs and video recordings, and through the act of thickening creating a description that represents interaction in coherent detail and makes it available to analysis [12].

Thus, while the record and replay tools described here provide the ethnographer with moment-by-moment access to video and to textual and graphical renderings of system records, the ethnographer must still turn to separate applications (notebook, word processor, spreadsheet, etc.) to create the ethnographic description itself. The links between record and replay tools and these different applications remains awkward and laborious. Nevertheless, the ongoing development of record and replay tools will rely on designers responding to and supporting the 'art and craft' of ethnographic data production and analysis.

Key to this achievement is the distinction between system time and interaction time and the need support the latter. Simply put, digital records of interaction in ubiquitous computing environments represent the logged order of events: represent when a text or audio message, for example, enters the system. However, when an event enters the system is not the same as when it enters interaction. Consider mobile phone use, by way of clarification: when a voicemail or text message is logged by the system is not the same as when the mail or message is acted upon - that may not be until sometime later, if at all. There is, then, a distinct difference between the logged order of events and the interactional order of events. Furthermore, the ethnographer's 'art and craft' is firmly oriented to unpacking the interactional order.

The logged order of events offers a beguiling representation of the order of interaction but it is a reified order. There is then a pressing need to develop representational tools that enable ethnographers to describe the interactional order of events as it observably and reportably unfolds, where the observability and reportability of the matter is determined by external resources such as video gathered by the ethnographer and where internal resources may be manipulated to be brought into accord with what it is that users visibly do. Thus, and for example, the ethnographer should be able to manipulate system logs to place such things as text and audio messages in a temporally and sequentially ordered description that reflects the position where logged events are responded to and visibly enter interaction.

All of the issues outlined above represent the focus of our current and future work. While a great deal of work is yet to be done, our experiences of record and replay have already added value to our understanding of interaction in ubiquitous environments - for details and analyses see [1, 2, $3,4,5,6,7,9,10,11,14]$ - and we think that much more is to be had by developing the approach.

\section{CONCLUSION}

We have been concerned in this paper to respond to the challenges facing ethnographic studies of ubiquitous computing applications in the wild, which arise from the invisible and fragmented character of many aspects of interaction in ubiquitous environments. Practically speaking, the ethnographer can only be in one place at one time and interaction in ubiquitous computing environments is distributed, mobile, mediated through small displays and virtual models alike, and conducted through different interaction mechanisms. There is a strong need to enhance observation in these environments, making the invisible visible and reconciling the fragments to permit coherent description. Our approach to this has been to augment the ubiquitous applications used in interaction to make a novel variety of system recordings that enable invisible and fragmented features of interaction to be replayed alongside one another and alongside resources gathered from outside the digital environment, especially video.

While it might be argued that we are not so much engaged in ethnography as a data gathering exercise, we remind the reader that data is not a natural object which may be readily harvested, but a socially and materially constructed object produced for particular purposes. The purpose here has been to articulate an embryonic range of tools that enable 
ethnographers working in an established interdisciplinary tradition to evaluate ubiquitous computing environments, and to encourage by example the broader adoption of this approach. Our early prototypes have already enabled us to make significant contributions to the understanding of interaction in ubiquitous computing environments. Furthermore, our experiences of using these prototypes have highlighted a range of challenges for the continued development of record and replay tools. Future work will involve refining this approach and these tools so that ethnographers are better equipped to study ubiquitous computing as it increasingly emerges from the laboratory and into our everyday lives.

\section{ACKNOWLEDGMENTS}

This research was funded by the EPSRC Equator IRCwww.equator.ac.uk - and the ESRC eSocial Science Research Node DReSS - www.ncess.ac.uk/nodes/ digitalrecord/

\section{REFERENCES}

1. Barkhuus, L. et al. (2005) "Picking pockets on the lawn”, Proc. of UbiComp '05, Tokyo: Springer.

2. Benford, S. et al. (2003) "Coping with uncertainty in a location-based game", IEEE Pervasive Computing, vol. 2 (3), pp. 34-41.

3. Benford, S. et al. (2004) "Uncle Roy All Around You", Proc. of ACE '04, Singapore: ACM.

4. Benford, S. et al. (2004) "The error of our ways", Proc. of UbiComp '04, pp. 70-87, Nottingham, UK: Springer.

5. Benford, S. et al. (2005) "Can You See Me Now?", to appear in ACM Transactions on Computer-Human Interaction.

6. Benford, S. et al. (2005) "Life on the edge", Proc. of CHI '05, pp. 721-730, Oregon: ACM.

7. Brown, B., MacColl, I., Chalmers, M., Galani, A., Randell, C. and Steed, A. (2003) "Lessons from the lighthouse”, Proc. of CHI '03, pp. 577-585, Florida: ACM.

8. Bittner, E. (1973) "Objectivity and realism in sociology", Phenomenological Sociology (ed. Psathas, G.), pp. 109-125, New York: John Wiley.

9. Crabtree, A., Benford, S., Rodden, T., Greenhalgh, C., Flintham, M., Anastasi, R., Drozd, A., Adams, M., Row-Farr, J., Tandavanitj, N. and Steed, A. (2004) "Orchestrating a mixed reality game 'on the ground", Proc. of CHI '04, pp. 391-398, Vienna: ACM.

10.Crabtree, A. (2004) "Design in the absence of practice", Proc. of DIS '04, pp. 59-68, Cambridge, MA: ACM.

11.Crabtree, A. (2005) "The social life of Uncle Roy", Equator Technical Report.

12.Crabtree, A. and Rouncefield, M. (2005) "Working with text $\operatorname{logs} "$, Proc. of $1^{\text {st }}$ International Conference on eSocial Science, Manchester: ESRC. www.mrl.nott.ac.uk/ axc/documents/eSS_2005.pdf
13.Czyzewski, M. (1994) "Reflexivity of actors versus the reflexivity of accounts", Theory, Culture and Society, vol. 11, pp. 161-168.

14.Flintham, M. et al. (2003) "Where on-line meets on-thestreets”, Proc. of CHI '03, pp. 569-576, Florida: ACM.

15.Garfinkel, H. (1967) Studies in Ethnomethodology, Englewood Cliffs, New Jersey: Prentice-Hall.

16.Garfinkel, H. and Sacks, H. (1970) "On formal structures of practical action", Theoretical Sociology (eds. M'Kinney, J.C. and Tiryakian, E.), pp. 160-193, New York: Apple-Century-Crofts.

17.Garfinkel, H. (2001) Ethnomethodology's Program: Working Out Durkheim's Aphorism (ed. Rawls, A.), Lanham, Maryland: Rowman and Littlefield.

18.Geertz, C. (1973) The Interpretation of Cultures, New York: Basic Books.

19.Hilbert, D. and Redmiles, D. (2000) "Extracting usability information from user interface events", $A C M$ Computing Surveys, vol. 32 (4), pp. 384-421.

20.Hughes, J., Randall, D. and Shapiro, D. (1992) "Faltering from ethnography to design", Proc. of CSCW '92, pp. 115-122, Toronto: ACM.

21.Ivory, M. and Hearst, M. (2001) "The state of the art in automating usability evaluation", ACM Computing Surveys, vol. 33 (4), pp. 470-516.

22.Kort, J. and de Poot, H. (2005) "Usage analysis: combining logging and qualitative methods", Proc. of CHI '05, pp. 2121-2122, Vienna: ACM.

23.Lyotard, J-F. (1984) "The post modern condition", Theory and History of Literature, vol. 10, Minneapolis: University of Minnesota Press.

24.Macbeth, D. (2001) “On 'reflexivity' in qualitative research: two readings, and a third", Qualitative Inquiry, vol. 7, pp. 35-68.

25.Mackay, W. and Beaudouin-Lafon, M. (1998) "DIVA", Proc. of CHI '98, pp. 416-423, Los Angeles: ACM.

26.Ryle, G. (1971) "The thinking of thoughts", University Lectures No. 18, University of Saskatchewan: Canada.

27.Sacks, H. (1992) "The baby cried. The mommy picked it up.", Lectures on Conversation (ed. Jefferson, G.), pp. 236-242, Oxford: Blackwell.

28.Sharrock, W. and Anderson, R. (1991) "Epistemology", Ethnomethodology and the Human Sciences (ed. Button, G.), pp. 51-76, Cambridge: Cambridge University Press.

29.Suchman, L. (1987) Plans and Situated Action, Cambridge: CUP.

30.Tolmie, P., Pycock, J., Diggins, T., Maclean, A. and Karsenty, A. (2002) "Unremarkable computing", Proc. of CHI '02, pp. 399-406, Minneapolis: ACM.

31.Woodruff, A., Szymanski, M., Grinter, R. and Aoki, P. (2002) "Practical strategies for integrating a conversation analyst in an iterative design process", Proc. of DIS '02, pp. 255-264, London: ACM. 
